\title{
CHARACTERISATION OF A TRACK STRUCTURE IMAGING DETECTOR
}

\author{
M. Casiraghi1,*, V. A. Bashkirov ${ }^{2}$, R. F. Hurley ${ }^{2}$ and R. W. Schulte ${ }^{2}$ \\ ${ }^{1}$ Center for Proton Therapy, Paul Scherrer Institut, Villigen, PSI 5232, Switzerland \\ ${ }^{2}$ Department of Basic Sciences, Loma Linda University, 11175 Campus Street, Loma Linda, CA 92350, USA
}

*Corresponding author: margherita.casiraghi@psi.ch

\begin{abstract}
The spatial distribution of radiation-induced ionisations in sub-cellular structures plays an important role in the initial formation of radiation damage to biological tissues. Using the nanodosimetry approach, physical characteristics of the track structure can be measured and correlated to DNA damage. In this work, a novel nanodosimeter is presented, which detects positive ions produced by radiation interacting with a gas-sensitive volume in order to obtain a high resolution image of the radiation track structure. The characterisation of the detector prototype was performed and different configurations of the device were tested by varying the detector cathode material and the working gas. Preliminary results show that the ionisation cluster size distribution can be obtained with this approach. Further work is planned to improve the detector efficiency in order to register the complete three-dimensional track structure of ionising radiation.
\end{abstract}

\section{INTRODUCTION}

Ionisation patterns on the nanometre scale have a fundamental role in the initial formation of biological damage produced by radiation. Complex DNA damage has been proven to correlate with irreparable radiation-induced lesions to DNA. However, experimental data of ionisation yield in condensed DNA material or liquid water are not directly measurable with the currently available techniques. To obviate this problem, a common approach is to measure the number of ionisations per primary particle produced in sensitive volumes (SVs) of gas, i.e. the ionisation cluster size distribution (ICSD). Under low-pressure conditions, these volumes are assumed to simulate water-equivalent nanometre-size volumes ${ }^{(1)}$.

Only few nanodosimeter prototypes are currently available. These include the ion-counter nanodosimeter and the jet counter, which register single ions produced in low-pressure gas. A different approach is used in the track-nanodosimetric counter, a single electron counter developed to measure the ICSD at different distances from the primary particle path. All of these detectors require sophisticated pumping equipment and careful alignment to low-intensity particle beams ${ }^{(2)}$.

In this work, the characterisation of a new generation gaseous detector has been performed, following up previously reported measurements ${ }^{(3)}$. This compact and relatively inexpensive nanodosimeter will allow a complete three-dimensional reconstruction of radiation track segments with single-ion resolution. With this experimental approach, the nanodosimetric characterisation of the radiation quality of unknown and mixed radiation fields can be obtained. Moreover, by superimposing the ionisation event pattern on nanometric SVs, the ICSD produced in the vicinity of DNA-like targets can be measured. Simulations of the detector applications are presented. The possibility to use measured quantities as input to radiobiological models that predict clustered DNA damage is also mentioned.

\section{MATERIALS AND METHODS}

\section{The detector operational principle}

The operational principle of the detector was previously presented in Bashkirov et al. ${ }^{(4)}$. The detector is a combination of a hole-type micro-pattern detector, such as gas electron multipliers (GEM), and a resistive plate chamber (RPC) operating at reverse polarity in low-pressure gas.

The detector design is shown in Figure 1. The main component is a 3-mm thick dielectric board, in which a two-dimensional array of sub-millimeter diameter cylindrical holes (cells) is created by means of printed circuit board (PCB) technology. A resistive cathode, in contact with the bottom side of the PCB is connected to a power supply which provides high voltage (HV) of the order of hundreds of volts. Positive ions, produced by charged particles traversing the working gas, drift towards the detector plane under an electric field $E_{\mathrm{d}}$ of the order of tens of $\mathrm{V} \mathrm{cm}^{-1}$. The drifting ions are focused into the cells and accelerated by the electric field $\left(E_{\mathrm{a}}\right)$. Operating in a gas pressure of the order of hundreds of Pascals, the reduced electric field inside each cell is of the order of $10 \mathrm{~V} \mathrm{~cm}^{-1} \cdot \mathrm{Pa}$. Under these conditions, the cross section for the ioninduced impact ionisation increases substantially and ionisation probability close to $100 \%$ can be obtained for a cell height of the order of a few millimetres ${ }^{(5)}$. The secondary electrons produced in this interaction process initiate an avalanche propagating towards the top of the cell with a gain of the order of $10^{8}$. The 


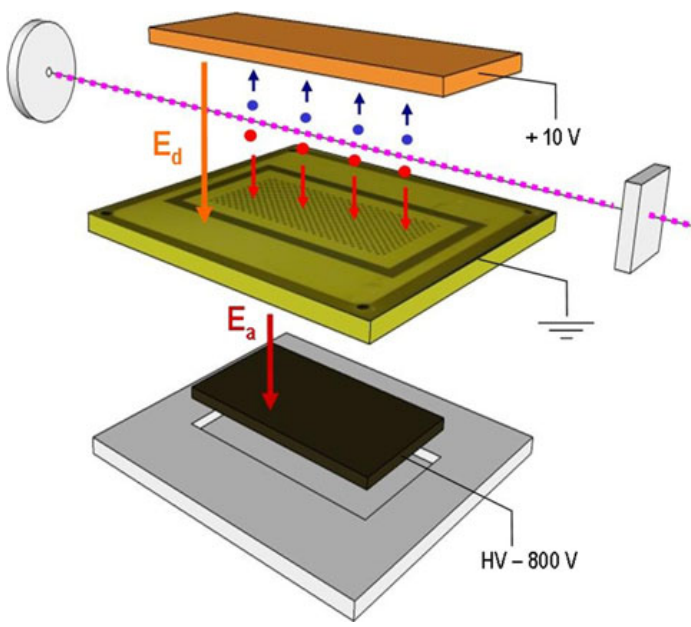

Figure 1. Sketch of the detector design.

avalanche is confined in time due to the high-volume resistivity of the cathode and it is spatially confined by the dielectric walls. As a consequence, each cell element operates as an independent Geiger counter. The top side of the PCB provides three readout strip layers: two orthogonal strip series, along each row and column of the cell array, respectively, and a third strip series with diagonal orientation (Figure 2). With this readout configuration, the position of the fired cell can be reconstructed. By registering positive ions rather than electrons, the diffusion is minimised and a series of two-dimensional time projections of the initial ionisation positions can be obtained. Additionally, the complete three-dimensional track image can be reconstructed by measuring the ion drift time.

\section{Experimental set-up}

Two versions of the detector prototype were assembled combining a PCB with a common top readout electrode with two different cathode types, a high resistivity glass and a semiconductor glass. The prototype was installed into a low-gas-pressure chamber providing a controlled gas environment. The chamber was filled with propane with a pressure value ranging from 133 to $667 \mathrm{~Pa}$. This allows a simulated tissue-equivalent $\mathrm{SV}$ of the order of a few tens of $\mu \mathrm{m}^{3}$. The operation with working gases as argon, nitrogen, and air was also tested. As primary radiation field, a ${ }^{241} \mathrm{Am}$ alpha source combined with a 2-mm diameter collimator was used. A silicon photodiode connected to a charge sensitive preamplifier was placed opposite to the source to measure the alpha rate and providing the trigger for data acquisition. Both the array detector signal and the trigger signal were sent to the data acquisition system. A field-programmable gate array (FPGA) was

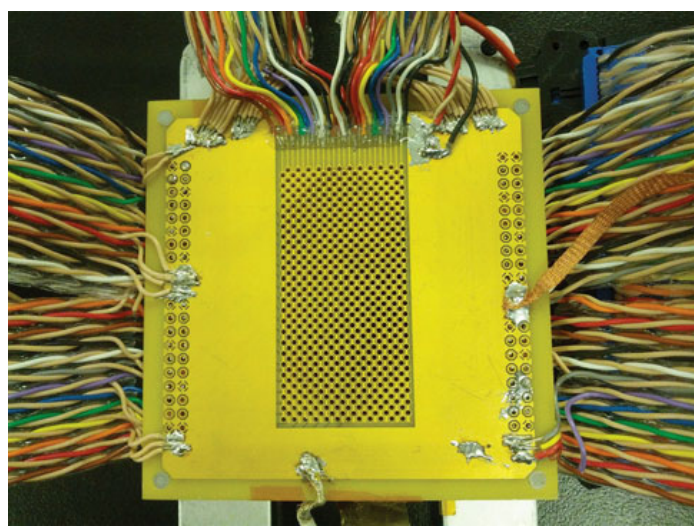

Figure 2. Assembled detector connected to $3 \times 24$ channel readout electronics.

used to measure the distribution of the number of pulses per trigger and the distribution of the pulse arrival time with respect to the trigger.

The measurements were performed applying a HV in the range of 650-850 $\mathrm{V}$ and a drift field in the range of $5-10 \mathrm{~V} \mathrm{~cm}^{-1}$ depending upon the working gas pressure.

\section{RESULTS}

\section{Detector characterisation}

In Figure 3(a) the detector output registered on the oscilloscope for alpha tracks in a SV of propane at a pressure of $400 \mathrm{~Pa}$ is shown. The signal consists of a series of negative pulses with amplitude varying from 2 to $8 \mathrm{mV}$ and duration of roughly $500 \mathrm{~ns}$. Each pulse corresponds to an average avalanche electron charge of the order of tens of $\mathrm{pC}$ that is generated in each individual cell of the two-dimensional array. The detector response using different working gases is shown in Figure $3(b-d)$. In the case of argon, the output signal is a combination of a series of negative short pulses and a long duration negative signal. The number of short pulses per alpha particle decreases with pressure. Below $400 \mathrm{~Pa}$, the short pulses disappear and a long duration signal, of $2 \mathrm{mV}$ amplitude, remains. Explanation of this behaviour is still under investigation. For both nitrogen and air, the signal corresponding to the alpha particle track is a long negative signal of about $40 \mu$ s duration. Such a signal can be due to the electronegativity of the two gases causing electron attachment forming slowly moving negative ions in the detector cells.

Figure 4 shows experimental distributions of detected ions per primary particle measured as detector counts per trigger. The dashed line and the solid line represent the distribution measured using the high resistivity and semiconductor cathode, respectively. 

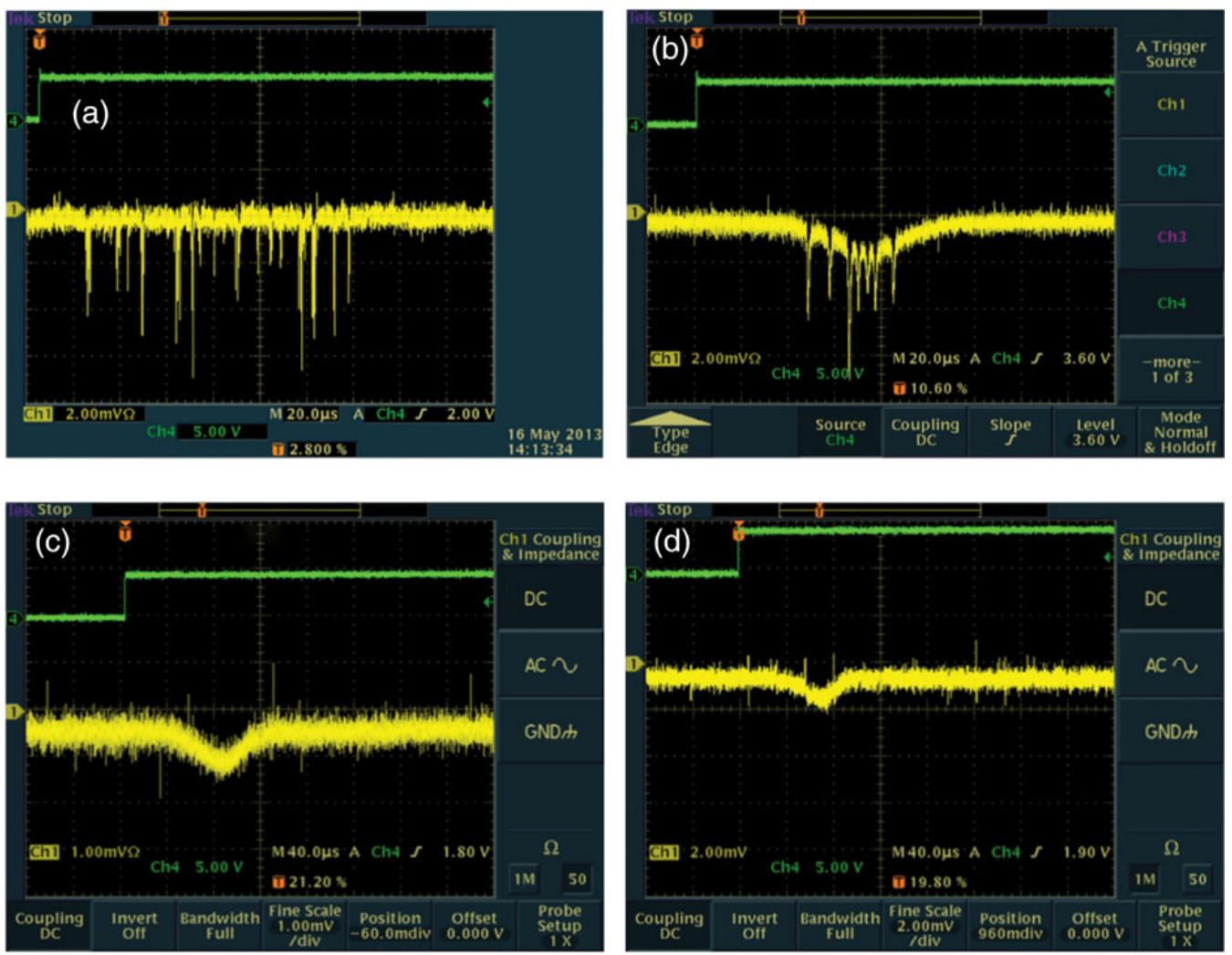

Figure 3. Detector response to different working gases: (a) propane, (b) argon, (c) nitrogen and (d) air. Channel 1: detector signal, channel 2: trigger signal.

Larger ion detection efficiency is observed when the semiconductor cathode is used due to a faster cathode recharge time.

The ideal detector response to $4.6 \mathrm{MeV}$ alphas in $400 \mathrm{~Pa}$ of propane was simulated with Geant4.9 Monte Carlo code ${ }^{(6)}$. A mean number of ionisations equal to 1655 was calculated from the simulated ICSD (Figure 5, dashed line plot). This value was compared with the measured mean number of ionisations per alpha particle obtained from the experimental distributions in Figure 4 in order to estimate the detector efficiency. A detector efficiency of 1 and $2 \%$ was estimated for the detector assembled with the resistive and semiconductor cathode, respectively.

In Figure 5 the comparison of the ideal ICSD and the ICSD simulated including the detector geometrical efficiency is shown. It can be observed that up to $78 \%$ of the signal is lost due to the geometrical factor. In the simulation, the worst case scenario is considered since the accelerating field which focuses the ions into the cells is neglected. However, the detector ion collection efficiency can be additionally improved by optimising the diameter and pitch of the
PCB cell array and by using the readout strip layers as elements of ion optics to provide additional focusing of the ions into the cells.

The detector dead-time due to the recovery of the accelerating field inside the fired cell is an additional cause for the low detection efficiency. Assembling the detector with a semiconductor cathode causes a decrease of the dead-time. However, this parameter can be additionally reduced by using slightly conductive PCB materials. This will allow a fast removal of the ions produced in the avalanche generated inside the cell that can diminish $E_{\mathrm{a}}$. Additionally, at the moment, the detector performance at pressure values below $200 \mathrm{~Pa}$ is affected by spurious discharges caused by HV components exposed to low pressure gas. To solve this problem, the design of an appropriate cathode insulation system is under way.

\section{Random sampling of radiation tracks as input for radiobiological models}

Monte Carlo simulations were performed in order to investigate possible applications of the detector to study 


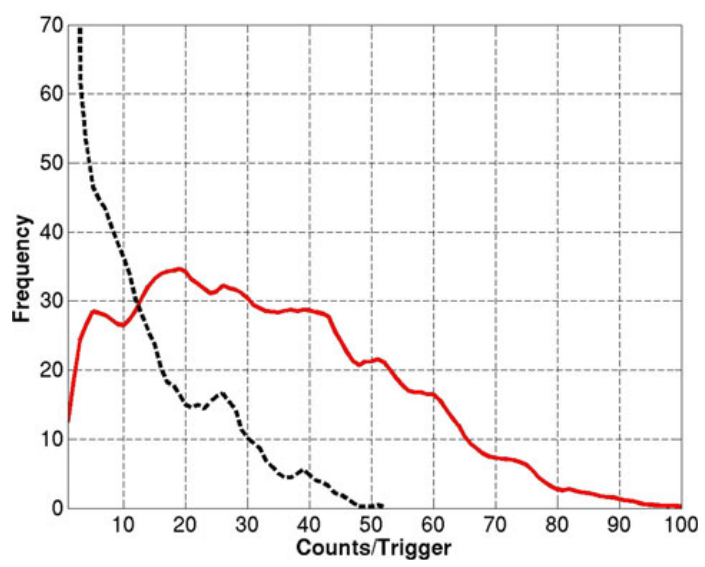

Figure 4. Distribution of the number of ionisations per trigger registered with the detector assembled with the high-resistivity cathode (dashed line, mean counts/trigger $=14$ ) and the semiconductor glass (solid line, mean counts/trigger $=32$ ). $p=400 \mathrm{~Pa}, \mathrm{HV}=-800 \mathrm{~V}, E_{\mathrm{d}}=10 \mathrm{~V} \mathrm{~cm}^{-1}$. With kind permission of The European Physical Journal (EPJ) ${ }^{(3)}$.



Figure 5. Simulation of the ICSD produced by $4.6 \mathrm{MeV}$ alphas in a volume of $5 \times 2 \times 1 \mathrm{~cm}^{3}$ of $400 \mathrm{~Pa}$ propane. Ideal detector response (dashed line). Detector response taking the geometrical efficiency into account (solid line). With kind permission of The European Physical Journal (EPJ) ${ }^{(3)}$.

the relation between the spatial distribution of ionisation events and the biological effect of radiation.

Tracks of $800 \mathrm{keV}$ protons produced in propane and water were simulated using the PTra code ${ }^{(7)}$ and Geant4-DNA ${ }^{(8)}$, respectively. As shown in Figure 6, the obtained tracks were sampled with randomly oriented cylindrical SVs assumed to represent DNA strands of roughly 50 base pairs. In Figure 7, the ICSDs simulated in propane at $133 \mathrm{~Pa}$ and water are

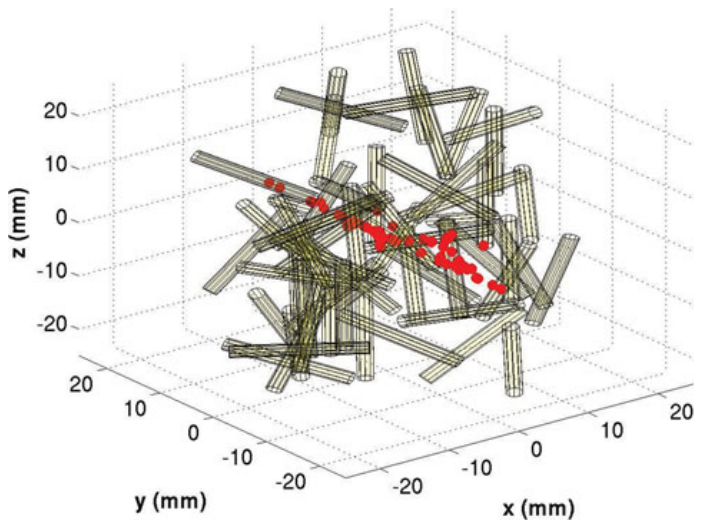

Figure 6. Simulation of an $800 \mathrm{keV}$ proton track in propane at $133 \mathrm{~Pa}$. The configuration of the randomly placed cylindrical SVs is also shown. SV dimensions are as follows: $D=0.3 \mathrm{~mm}$ and $h=2.5 \mathrm{~mm}$ equivalent to 2 and $16 \mathrm{~nm}$ in water, respectively.

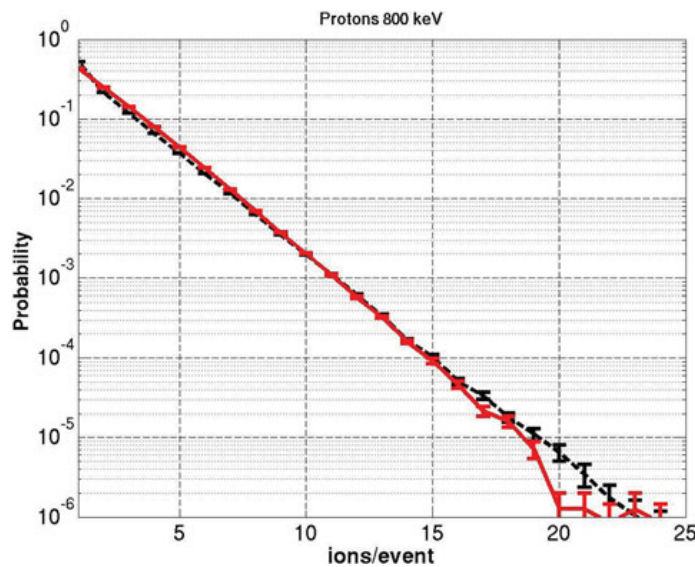

Figure 7. Simulated ICSDs produced by $800 \mathrm{keV}$ protons in cylindrical SVs of water (dashed line) and propane (solid line).

compared. For the simulations in water, cylindrical SVs of $2 \mathrm{~nm}$ of diameter and $16 \mathrm{~nm}$ height were used. The equivalent SV dimensions in propane were calculated applying a scaling factor depending on gas density and the ratio of the ionisation mean free path of the primaries in the two media. The obtained curves are in good agreement above a probability of $10^{-5}$. This result indicates that information about interaction of radiation with biological tissue may be estimated performing measurements in gas with the track imaging detector. The measured nanodosimetric quantities can then be used as input to models that predict the yields of simple and complex double strand breaks of DNA as proposed by Schulte et $a l .{ }^{(9)}$. 


\section{CONCLUSIONS}

Preliminary results show that the detector principle proposed to detect ionisation events produced in low pressure gas with a single-ion resolution is feasible. ICSDs of primary radiation interacting with the SV can be measured with this device. At the moment, propane seems to be the most appropriate working gas to obtain a single-ion resolution signal but the use of other working gases is under study. Comparison of measurements to simulations shows rather low detection efficiency, in the order of a few per cent. However, the detector is still in its early stages and several measures to improve its performance are under study.

Simulations performed to investigate the possible detector applications show that the three-dimensional radiation track image obtained in gas can be analyzed in order to obtain information about the ionisation patterns produced in biologically relevant targets.

\section{FUNDING}

This work is supported by the European ANDANTE Project funded by Euratom FP7. The PTra code was developed by Bernd Grosswendt and its latest version kindly provided by Marion Bug at PTB.

\section{REFERENCES}

1. Grosswendt, B. Nanodosimetry, from radiation physics to radiation biology. Radiat. Prot. Dosim. 115, 1-9 (2005).

2. Schulte, R. W. Nanodosimetry: principle and current status. AIP Conf. Proc. 1345, 249-261 (2011).

3. Casiraghi, M., Bashkirov, V., Hurley, F. and Schulte, R. A novel approach to study radiation track structure with nanometer-equivalent resolution. Eur. Phys J. D 68, 111 (2014).

4. Bashkirov, V. A., Hurley, R. F. and Schulte, R. W. A novel detector for $2 D$ ion detection in low-pressure gas and its applications. In: NSS/MIC Conference Record, IEEE, pp. 694-698, (2009).

5. Garty, G., Shchemelinin, S., Breskin, A., Chechik, R., Assaf, G., Orion, I., Bashkirov, V., Schulte, R. and Grosswendt, B. The performance of a novel ion-counting nanodosimeter. Nucl. Instr. Meth. A 491, 212-235 (2002).

6. GEANT4 Collaboration. GEANT4-a simulation toolkit. Nucl. Instrum. Methods Phys. Res. 506, 250-303 (2003).

7. Grosswendt, B. Formation of ionization clusters in nanometric structures of propane-based tissue-equivalent gas or liquid water by electrons and alpha-particles. Radiat. Environ. Biophys. 41, 103-112 (2002).

8. Incerti, S. et al. Comparison of Geant4 very low energy cross section models with experimental data in water. Med. Phys. 37, 4692-4708 (2010).

9. Schulte, R. W., Wroe, A. J., Bashkirov, V. A., Garty, G. Y., Breskin, A., Chechik, R., Shchemelinin, S., Gargioni, E., Grosswendt, B. and Rosenfeld, A. B. Nanodosimetrybased quality factors for radiation protection in space detection in low-pressure gas and its applications. Z. Med. Phys. 18, 286-296 (2008). 